\title{
Building a multipurpose insertional mutant library for forward and reverse genetics in Chlamydomonas
}

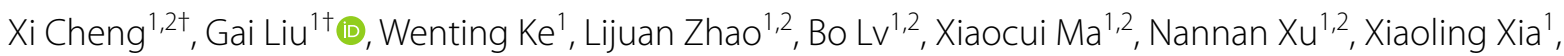 \\ Xuan Deng ${ }^{1}$, Chunlei Zheng ${ }^{3}$ and Kaiyao Huang ${ }^{1 *}$
}

\begin{abstract}
Background: The unicellular green alga, Chlamydomonas reinhardtii, is a classic model for studying flagella and biofuel. However, precise gene editing, such as Clustered Regularly Interspaced Short Palindromic Repeats (CRISPR) and CRISPR-associated protein (Cas9) system, is not widely used in this organism. Screening of random insertional mutant libraries by polymerase chain reaction provides an alternate strategy to obtain null mutants of individual gene. But building, screening, and maintaining such a library was time-consuming and expensive.

Results: By selecting a suitable parental strain, keeping individual mutants using the agar plate, and designing an insertion cassette-specific primer for library screening, we successfully generated and maintained 150,000 insertional mutants of Chlamydomonas, which was used for both reverse and forward genetics analysis. We obtained 26 individual mutants corresponding to 20 genes and identified 967 motility-defect mutants including 10 mutants with defective accumulation of intraflagellar transport complex at the basal body. We also obtained 929 mutants defective in oil droplet assembly after nitrogen deprivation. Furthermore, a new insertion cassette with splicing donor sequences at both ends was also constructed, which increased the efficiency of gene interruption.
\end{abstract}

Conclusion: In summary, this library provides a multifunctional platform both for obtaining mutants of interested genes and for screening of mutants with specific phenotype.

Keywords: Chlamydomonas, Insertional mutants, Mutant library, Flagella, Intraflagellar transport, Oil droplet

\section{Background}

Chlamydomonas reinhardtii is a unicellular green alga that shares organelles with both higher plants and animals [1]. This organism performs photosynthesis via cup-shaped chloroplasts, moves toward nutrients and light using a pair of flagella/cilia, and accumulates oil droplets in response to nitrogen starvation [2]. So Chlamydomonas is recognized as an important model for studying photosynthesis, flagella/cilia, and stress response [3, 4]. Recently, It has been acknowledged as "green yeast" for the study of biofuels such as biodiesel

\footnotetext{
*Correspondence: huangky@ihb.ac.cn

${ }^{+}$Xi Cheng and Gai Liu are Co-first authors

1 Key Laboratory of Algal Biology, Institute of Hydrobiology, Chinese

Academy of Sciences, Wuhan 430072, China

Full list of author information is available at the end of the article
}

and hydrogen, and has been used as a biofactory for the production of antibodies and biopharmaceuticals $[5,6]$.

Classic and modern genetic manipulation techniques have been established in the last 60 years since Gilbert Morgan Smith isolated and provided the first C. reinhardtii strain to the scientific community [7]. Ultraviolet (UV) light [8], ethyl methanesulfonate (EMS) [9] and $N$-methyl- $N^{\prime}$-nitro- $N$-nitrosoguanidine (MNNG) [10, 11] was widely used for mutagenesis and generated many useful mutants. For example, the genes involved in photosystem-II and photosynthetic electron transport were first characterized in Paul Levine's laboratory by screening UV-induced acetate auxotrophy mutants $[8,12]$. Cell wall-deficient mutants have been generated by MNNG mutagenesis and isolated under stereomicroscopy. These mutants have been widely used for gene transformation 
using glass beads [13]. Remarkably, temperature-sensitive flagellar mutants generated by MNNG mutagenesis are motile at permissive temperatures, but become immotile at restrictive temperatures, and have been used to identify the components of intraflagellar transport (IFT) [11, 14].

Transformation or introducing a foreign gene in the genome is a prerequisite of modern genetics. Several methods have been developed to transform DNA fragments into the nuclear genome of Chlamydomonas. In addition to glass beads mentioned above, other techniques include electroporation, biolistic transformation and biotransformation by Agrobacterium tumefaciens are also used in Chlamydomonas [15-17]. Biolistic transformation can also be used to transform DNA into the chloroplast and mitochondria of Chlamydomonas [18, 19]. Meanwhile, various transformation markers have been developed. These include auxotrophic markers such as argininosuccinate lyase ( $A R G 7)$ and nitrate reductase (NIT1), which encode enzymes that complement metabolic deficiencies [15, 20], as well as antibiotic markers such as aphVII, aphVIII, and ble, which provide resistance to hygromycin, paromomycin and zeocin respectively [21-23]. Several novel markers have also been developed recently, such as herbicide resistance genes glyphosate acetyltransferase (GAT), protoporphyrinogen oxidase $(P P O)$, and phytoene desaturase $(P D S)$ and codon-optimized kanamycin-resistance gene nptII and tetracycline resistance gene tet $X$ [24-26].

Similar to other classical organism models, both forward and reverse genetics strategies are widely employed in the research of Chlamydomonas. Forward genetics study generally composed of four major steps: mutagenesis, phenotypic screening, mutation loci (gene) mapping, and phenotype rescue. Much progress has been made to optimize these four steps. At present, mutagenesis introduced by insertion of a DNA cassette into the genome is preferred [27-29]. The mutated gene can be identified using PCR map-based cloning, and whole genome sequencing [29-31]. The valuable Chlamydomonas mutant library screened by forward genetics could also be cryopreserved for further analysis [32]. Specific mutated phenotypes can be screened using high throughput methods such as fluorescence-activated cell sorting [33]. Phenotype rescue is relatively difficult, because the expressions of foreign genes are relatively low in Chlamydomonas [34]. However, this problem may be solved partially by modification of transgene sequences, such as codon optimization, incorporation of native promoters and introns, co-expression with antibiotic gene or luciferase, and use of a host strain defective in transgene silencing [34-36].
Several methods are also available for reverse genetic studies in Chlamydomonas. Target genes can be knocked down by using small interfering RNAs [37] and artificial miRNAs [38, 39]. Conditional knockdown of Chlamydomonas genes can be achieved by using inducible promoters such as nitrate-induced promoter NIT1, nickel-induced promoter $\mathrm{CYC6}$, and $\mathrm{CO}_{2}$-induced promoter CAH1 [40, 41]. However, the knockdown effects of RNAi [37] and artificial miRNAs [38] varies depending on genes and may disappear after several months. Gene knockout based on homologous recombination (HR) provides a great opportunity to study the function of non-lethal genes. Of note, is that the natural HR rate in Chlamydomonas is extremely low [42]. Several strategies have been described to increase the HR rate, such as use of single-stranded DNA, use of HR constructs coding an antibiotic marker in frame with the target gene, and induction of DNA breaks in the target gene via zincfinger nucleases [42, 43]. However, these methods are either limited to special genes or the procedures are tedious. Recently, gene editing using CRISPR and Cas9 has been reported in Chlamydomonas, but the efficiency is too low to be applied to any genes, especially when the phenotypes of mutated genes are unknown [44-46]. Thus, constructing a random insertional mutant library provides an alternative method to obtain the mutants of specific genes. This strategy has been successfully applied to construct a single-use insertion mutant library and obtained 45 individual mutants representing 37 different genes by screening the approximately 100,000 insertional mutants with PCR [29]. Most recently, an indexed, mapped mutant library was developed based on the highthroughput approach, in which the mutants were transferred by highly automatic robots and cryopreserved in liquid nitrogen. The insertion sites were determined by deep sequencing methods, Chlamydomonas MmeIbased insertion site Sequencing (ChlaMmeSeq) and Linear and Exponential Amplification of insertion site sequence coupled with Paired-end Sequencing (LEAPSeq) $[27,47]$. As a results, 37,000 mutants covering $73 \%$ of the Chlamydomonas genome were identified based on a deconvolution method [47]. However, construction and maintenance of such a library requires automatic robot technology, which is expensive for a small group. The library is mostly used for reverse genetics and more than half of the insertions lie in the untranslated region (UTR) [47]. Therefore, the need for improvement in construction, maintenance, and screening the library still remains.

In this study, a library of approximately 150,000 Chlamydomonas transformants was constructed and maintained with optimized procedures. Both reverse and forward genetics were performed to identify mutants 
of specific genes or mutants with specific phenotypes. We obtained 967 mutants with defective motility, 929 mutants with fewer oil droplets in response to nitrogen deprivation, and 26 individual mutants representing 20 different genes related to flagella, glycosylation, and starch metabolism. This library can also be used to screen mutants for any Chlamydomonas gene of interest or mutants with specific phenotype defects.

\section{Results}

\section{Optimizing methods for construction of the insertional library}

The genomic size of Chlamydomonas is about $111 \mathrm{Mb}$ and the genome encodes approximately 17,000 genes according to the prediction in Joint Genome Institute (JGI) version 5.5 of Chlamydomonas genome [1]. Since the integration of DNA fragment in the genome of Chlamydomonas is random, the minimum number of insertional mutants is 111,000 if one insertional mutation locating within any $1 \mathrm{~kb}$ of genome is required. Taking into account that the average size of Chlamydomonas genes is approximately $6 \mathrm{~kb}$, each gene can be hit about five times. However, to generate and maintain this quantity of mutants is challenging for an individual group. To optimize the use of this resource, we intended this library to be used not only for reverse genetics, but also forward genetics, such as screening mutants for flagella assembly and formation of oil droplets.

\section{Selection of the parental strain}

Intraflagellar transport (IFT) plays a key role in flagellar assembly and disassembly [4]. In order to obtain the mutants defective in IFT protein accumulation at the basal body, we generated an IFT46::YFP fusion construct using an endogenous promoter of IFT46 and transformed it into the flagella-less mutant ift46-1 (Fig. 1a). IFT46::YFP rescued the motility defect of ift46-1 and

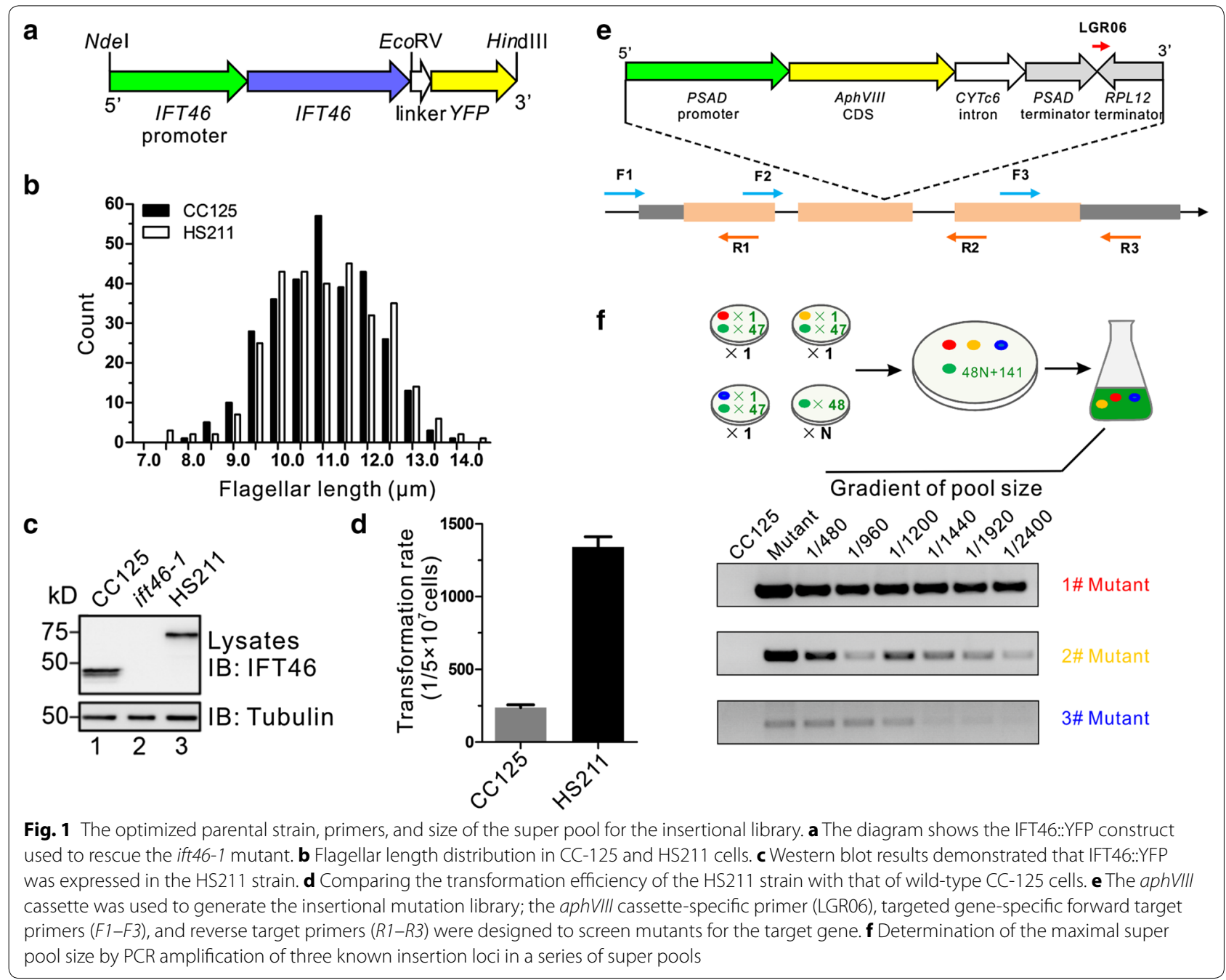


the distribution of flagellar length in the rescued strain was similar to the wild type, with an average length of $11.1 \mu \mathrm{m}$ (Fig. 1b). The rescued strain was designated as HS211. Western blot results demonstrated that IFT46 was expressed in CC-125 cells only and not in the ift461 mutant, while the fusion protein IFT46::YFP was detected in HS211 (Fig. 1c). These data suggest that the IFT46::YFP fusion protein functions as endogenous IFT46. Thus, HS211 could serve as a parental strain for screening of flagella assembly mutants and IFT basal body localization-defect mutants.

Next, we compared the transformation efficiency of HS211 with wild type strain. Since the aphVII gene conferring to hygromycin resistance was used to generate the HS211 strain, transformation efficiency of HS211 was tested using the aminoglycoside $3^{\prime}$-phosphotransferase (aphVIII) cassette as a selection marker. Using $5 \times 10^{7}$ cells for one transformation, an average of 1342 transformants were obtained in HS211, whereas only 236 were obtained in CC-125 cells. The transformation efficiency of HS211 was sixfold higher than that of the wild type, CC-125 (Fig. 1d). This indicates that one transformation using $30 \mathrm{ng}$ aphVIII in an electroporation cuvette generated almost 1400 transformants after 3 days, which suggests that only $\sim 100$ times transformations can generate enough mutants for construction of the library.

\section{Insertion cassette and screening primer design}

Besides serving as a selection marker for transformation, an ideal cassette for insertional mutagenesis can terminates transcription of the interrupted gene completely. Therefore, the $2.6 \mathrm{~kb} M l y \mathrm{I}$ digested fragment from plasmid pMJ013b was used, which contains the promoter and terminator of PSAD gene, the aphVIII gene, an intron from CYTc6, and an RBL12 terminator [27] (Fig. 1e). The intron was intended to enhance the expression of the $a p h$ VIII gene and two inverted terminators at the $3^{\prime} \mathrm{UTR}$ were designed to terminate gene transcription of interrupted gene, irrespective of the direction of insertion. The fragment was therefore, selected as the insertion cassette.

The principle behind PCR-based screening was that when an insertion occurred in a target gene, a PCR product can be obtained using an insertion cassette-specific primer and a target gene-specific primer [29]. Since GC content of the Chlamydomonas genome is approximately $60 \%$, the PCR product should be $<1 \mathrm{~kb}$ in order to be considered reliable. Therefore, the target gene-specific primers were designed in two directions, forward and reverse, at an interval of approximately $1.0 \mathrm{~kb}$. In order to cover every possible insertion events, the target gene-specific primers were designed from $500 \mathrm{bp}$ upstream of $5^{\prime} \mathrm{UTR}$ to the end of $3^{\prime} \mathrm{UTR}$ of any interested genes (Fig. 1e).
The insertion cassette-specific primer requires to be within the 200- to 500-bp region from the insertion cassette border [29]. This region not only ensures amplification specificity and efficiency, but also reduces the possibility of corruption of the annealing sites caused by recombination or endonucleolytic cleavage during transformation [27]. The primer previously used has been designed as per the aphVIII gene sequence or the endogenous sequence of the PSAD promoter and RBL12 terminator. The aphVIII gene in this $2.6-\mathrm{kb}$ cassette was approximately $700 \mathrm{bp}$ from the border, while the primers annealing to PSAD promoter and RBL12 terminator tended to generate non-specific amplification since there is another copy of these two genes in the genome. Therefore, we designed the cassette-specific primer LGR06, annealing to the junction between PSAD and RBL12 terminator, which was 273 bp near the border of insertion cassette. Compared with the cassette-specific primer LGL03, which anneals to the PSAD promoter, the sequence of LGR06 does not exist within the Chlamydomonas genome. PCR amplifications with LGR06 generate fewer non-specific products and a cleaner background using the library and HS211 genomic DNA as templates. This primer increased efficiency of library screening (Additional file 1: Fig. S1).

\section{Determination of the super pool size}

A super pool size up to approximately 1000 was adopted for mutants screening in the previously generated library [29]. Larger pool size can reduce labor cost significantly if PCR amplification sensitivity can be guaranteed. We identified the maximal size of the super pool for library screening by mixing three characterized insertional mutants with varying numbers of uncharacterized insertional mutants, to generate a series of super pools ranging from 480 to 2400 in size (Fig. 1f). PCR amplification of three different insertion loci indicated that two loci could be detected in super pool reached to 2, 400, another one only could be identified by PCR in super pool 1920, so we choose 1440 as the optimal size for super pool (Fig. 1f).

\section{Maintenance and replication of transformants}

Maintenance and replication of large-scale Chlamydomonas mutants is a time-consuming task. To overcome this problem, a single-use library of up to 100,000 transformants was generated [29]. Alternatively, a library consisting of 18,334 transformants was cultured in 96- or 384- $\mu$ l plates and cryopreserved with automatic robots operated under highly sterile conditions [47]. We intended to save all transformants on an agar plate and therefore devised an optimal economic 
strategy. After electroporation, the transformants grew on solid tris-acetate-phosphate (TAP) plates under low light for 1 week (Fig. 2a), then each clone was picked up with a toothpick and applied along a line across the agar surface, where the clone propagated to the entire length of the line within a week. Thus, 48 lines were obtained (Fig. 2b1). This method not only guaranteed enough cell count for further analysis and reduced the duration for replication of the library, but also reduced loss of transformants due to contamination, since only 48 colonies would be lost in case a plate was contaminated. After a year, the livability of our library was approximately $97.8 \%$, and the contamination rate was lower than $3 \%$.

\section{Isolation of the super pool DNA}

Successful PCR amplification of insertion loci depends on quality of DNA templates from each super library. The library should contain sufficient DNA for thousands of PCR runs and also contain similar amount of genomic DNA of each mutant. To achieve this goal, we inoculated 1440 transformants from the basic pool onto $15-\mathrm{cm}$ sized plates by drawing a shorter colony line (Fig. 2b2), such that the similar amount of cells from each clone could be guaranteed. All the transformants grown into visible clones of similar size within several days, then the clones were scraped and resuspended in liquid TAP medium, forming a cell culture with concentration $>5 \times 10^{6}$ cells/ $\mathrm{ml}$. After the cell reached stationary phase under constant light, genomic DNA was isolated using the phenol/chloroform method [48]. Generally, about $250 \mu \mathrm{g}$ genomic DNA can be obtained from a single super pool library preparation, which is adequate to screen 2500 pairs of primers.

\section{Screening the mutants based on reverse genetics strategy}

We planned to screen insertional mutants of 61 genes of interest, which requires more than 600 target genespecific primers to screen 104 super pool libraries (Additional file 2: Table S1 and Additional file 3: Table S2). This means that at least 62,000 PCR reactions and 630 PCR runs need to be performed. To save time and cost, we optimized several aspects of the PCR screening strategy. First, the super pool libraries were divided into two groups, each including 48 libraries. If a reliable mutant was identified in one group, the other group was skipped. Second, DNA templates were arrayed into a matrix consisting of 4 rows and 12 columns. All PCR components were premixed and the reaction buffers were prepared only with addition of different targetspecific primers. Thus, templates and buffers could be transferred into PCR microplates with a multichannel pipette (Fig. 2b4). Third, since it is generally accepted that insertional mutagenesis near the start codon tends to induce a complete knock-out effect, the screening process for each target gene was initiated with the first six target primers either forward or reverse from the $5^{\prime}$ UTR end. If a correct insertion was identified by sequencing the PCR product, the other primers and libraries were not screened (Fig. 2b5). As a result, the total number of PCR runs was largely reduced. Finally, the volume of each PCR was reduced to $20 \mu \mathrm{l}$ and the amount of Taq polymerase used per reaction was reduced from 2 units (supplier recommended) to 0.5 units, which improved amplification specificity per contra.

Once a correct insertion was identified from a super pool, the basic pools from this positive super pool were recovered for DNA isolation (Fig. 2b6). All 96 colonies from each basic pool were replicated onto a fresh 9-cm solid agar plate, allowing all transformants to recover to similar extents (Fig. 2b6). Then, cells from these plates were washed with $20 \mathrm{ml}$ TAP medium and genomic DNA from each basic pool was isolated using the phenol/chloroform method, but on a small scale (Fig. 2b6). Approximately $10 \mu \mathrm{g}$ basic library DNA was harvested, which was adequate for identification of positive transformants from the basic pool (Fig. 2b7). Once the positive basic pool was identified, all 96 transformants from the positive basic pool were inoculated separately, into liquid TAP medium contained in 96 microtiter plates. The cells were mixed together by line and row respectively, and colony PCR was performed. Positive results were obtained from both, the row and line mixtures as a positive transformant (Fig. 2b8). Finally, the positive transformant was duplicated from the original 9-cm solid agar plates for further analysis (Fig. 2b9).

\section{Screening flagellar assembly-defect mutants}

In addition to screening mutants for any gene of interest by reverse genetics, the library was also utilized to screen flagellar-defect mutants. Since the flagella of gametes are longer than those of vegetative cells [49], an aliquot of each clone was transferred into liquid nitrogen-deprived TAP (TAP-N) medium in 96-well microtiter plates for gametogenesis and screened for mutants with motility defects under a stereomicroscope (Fig. 2c1). We identified 967 motility-defect mutants (Fig. 2c2). Since motility defects may result from flagella loss or from the flagella being short or paralyzed, the flagellar length of these mutants was measured. We found that $66 \%$ of these mutants failed to assemble flagella, $11 \%$ showed shorter flagella, while the rest exhibited flagella with normal length, but were paralyzed. This suggests that most motility defects are caused by aberrations in flagella assembly (Fig. 3a). 


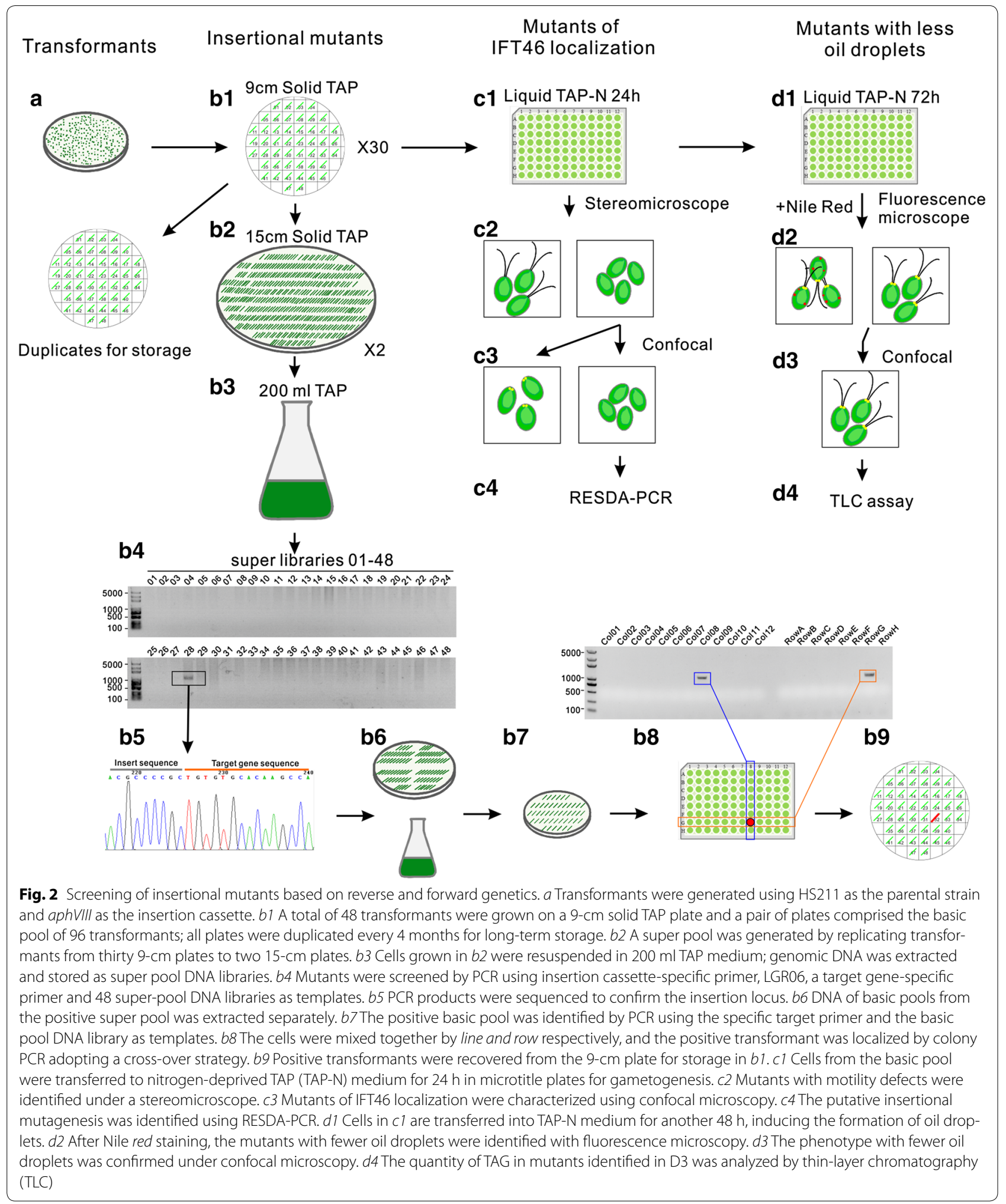

Because the IFT marker IFT46::YFP was introduced into the parental strain HS211, all motility-defect mutants with aberrant IFT distribution at the basal body were screened. In the wild type HS211, the puncta of IFT46::YFP were distributed along the flagella and IFT46::YFP accumulation was observed at the basal body 


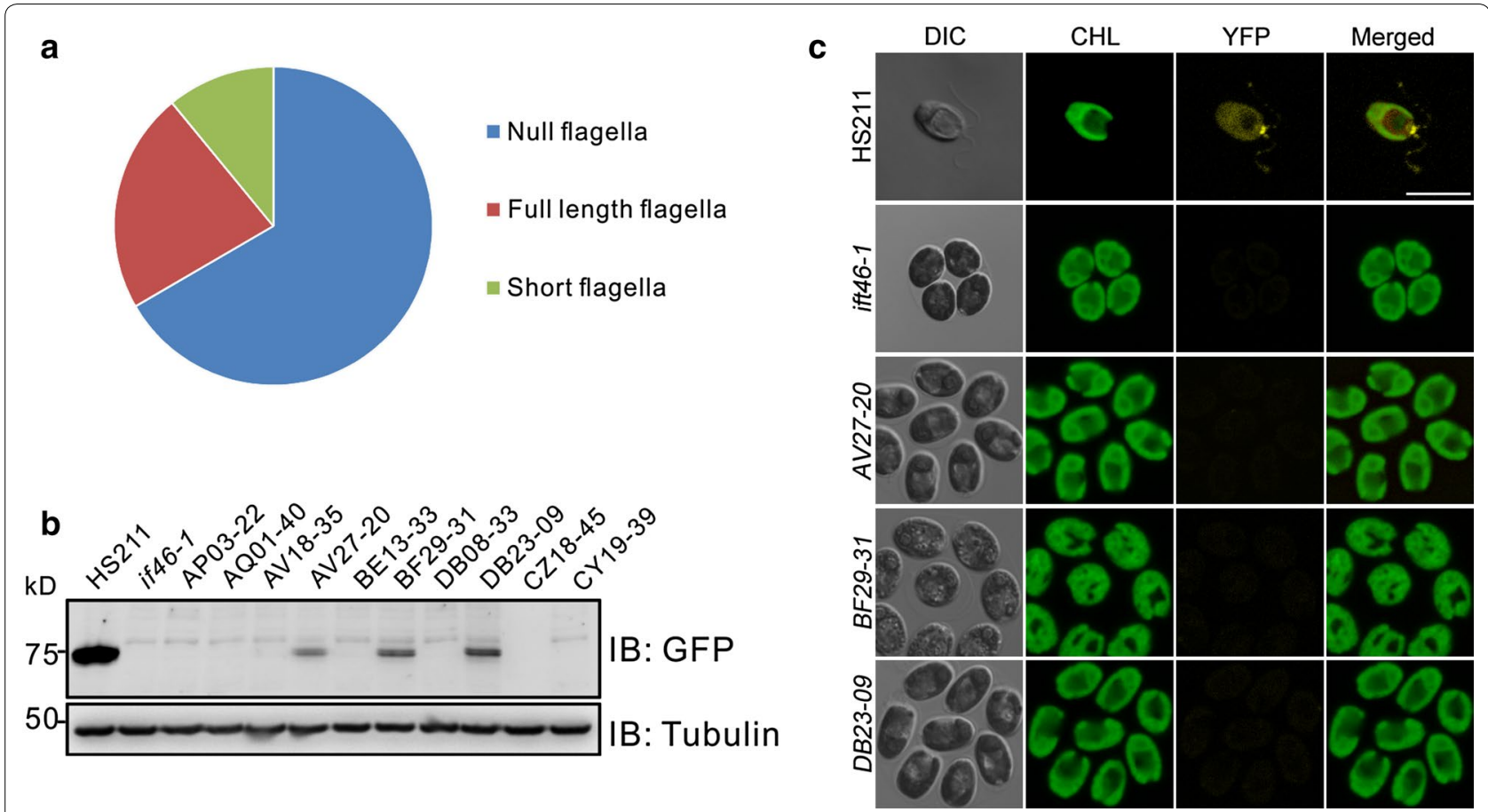

Fig. 3 Mutants with motility defects and abnormal IFT46 localization. a Flagellar motility defects resulted from null, short, or paralyzed flagella. b Expression profile of IFT46::YFP in HS211 and the mutants. c Fluorescence micrographs showing IFT46::YFP localized at the basal body in HS211 cells and disappeared in mutants. Scale bar $10 \mu \mathrm{m}$. CHL chlorophyll fluorescence

(Fig. 3c). In most of 638 flagella-less mutants, IFT46::YFP was concentrated at the basal body, while the fluorescence signal from some of these mutants was a little weaker. However, ten mutants showed similar phenotype with the ift46-1 mutant, lacking IFT46 accumulation at the basal body (Fig. 3c). The loss of IFT46::YFP signal was probably caused by knockout of IFT46::YFP in the parental strain, interference of IFT46 transcription, or regulation of IFT46 anchoring to basal body. To investigate this, IFT46::YFP expression in ten mutants was analyzed by western blot (Fig. 3b). IFT46::YFP proteins were absent in seven mutants, but were detected in the remaining three mutants, at reduced quantities compared to the parental strain HS211. This indicates that certain key elements regulating IFT46 anchorage to the basal body may have been knocked out in these three mutants. The flanking sequences of the insertion cassette in these mutants were amplified using restriction enzyme site-directed amplification (RESDA)-PCR in order to map the insertion loci (Table 1). In the mutant DB23-09, the cassette was inserted into the ninth intron of IFT88, which is required for the localization of IFT-B complex to the basal body [50]. The characterization of ift 88 mutant as exhibiting IFT46 distribution defect suggests that the screening strategy we used was reasonable. The other two insertion loci in mutants AV27-20 and BF29-31 were localized to genes Cre14.g633700, which is an uncharacterized protein with transmembrane domain, and Cre11.g467653, which is annotated as threonine-specific protein kinase. Nevertheless, further analyses are required to confirm their role in regulation of IFT46 accumulation at the basal body.

\section{Screening mutants with defective in oil droplet assembly} Chlamydomonas accumulates oil droplets in response to nitrogen deprivation [51]. Therefore, we used this library to screen oil droplet assembly mutants. The parental strains and mutants were cultured in TAP-N medium for $48 \mathrm{~h}$, and stained with Nile red. Oil droplets accumulated in the cell body were observed under a fluorescence microscope (Fig. 2d1). A total of 929 mutants with fewer oil droplets were characterized (Fig. 2d2); of these, 11 mutants were selected and observed under a confocal microscope (Fig. 2d3). The fluorescence intensities of Nile red in 5 mutants were decreased significantly as compared to the parental strain, representing a reduced number and size of oil droplets (Fig. 4a). Thin layer chromatography (TLC) indicated that 4 mutants synthesized less triacylglycerol (TAG) after nitrogen deprivation (Fig. 4b, c). The mutant of AP01-30 and DA06-10 only have $60 \%$ TAG compared the parental strains HS211. These data suggest that a large amount of mutants 
Table 1 The mutants lacking IFT46::YFP accumulation at the basal body

\begin{tabular}{|c|c|c|c|}
\hline Strain & Accession of inserted gene ${ }^{a}$ & Annotation & Insertion loci ${ }^{\mathbf{b}}$ \\
\hline AP03-22 & Cre11.g467717 & Nitrilase-related; carbon-nitrogen hydrolase & R, 5th intron \\
\hline AQ01-40 & Not identified & - & - \\
\hline AV18-35 & Between Cre14.g612250 and Cre14.g612226 & No annotation & Intergenic region \\
\hline AV27-20 & Cre14.g633700 & Uncharacterized conserved protein & $\mathrm{R}$, 4th intron \\
\hline BE13-33 & Not identified & - & - \\
\hline BF29-31 & Cre11.g467653 & Threonine-specific protein kinase & R, 2nd intron \\
\hline DB08-33 & Not identified & - & - \\
\hline DB23-09 & Cre07.g335750 & Intraflagellar transport protein 88 & F, 9th intron \\
\hline CZ18-45 & Not identified & - & - \\
\hline CY19-39 & Cre12.g554400 & No annotation & F, 1st exon \\
\hline
\end{tabular}

a Gene accession numbers were from Phytozome v10.3 C. reinhardtii website

b $\mathrm{F}$ and $\mathrm{R}$ indicate that the insertion cassettes have the same or opposite orientation as the targeted genes, respectively

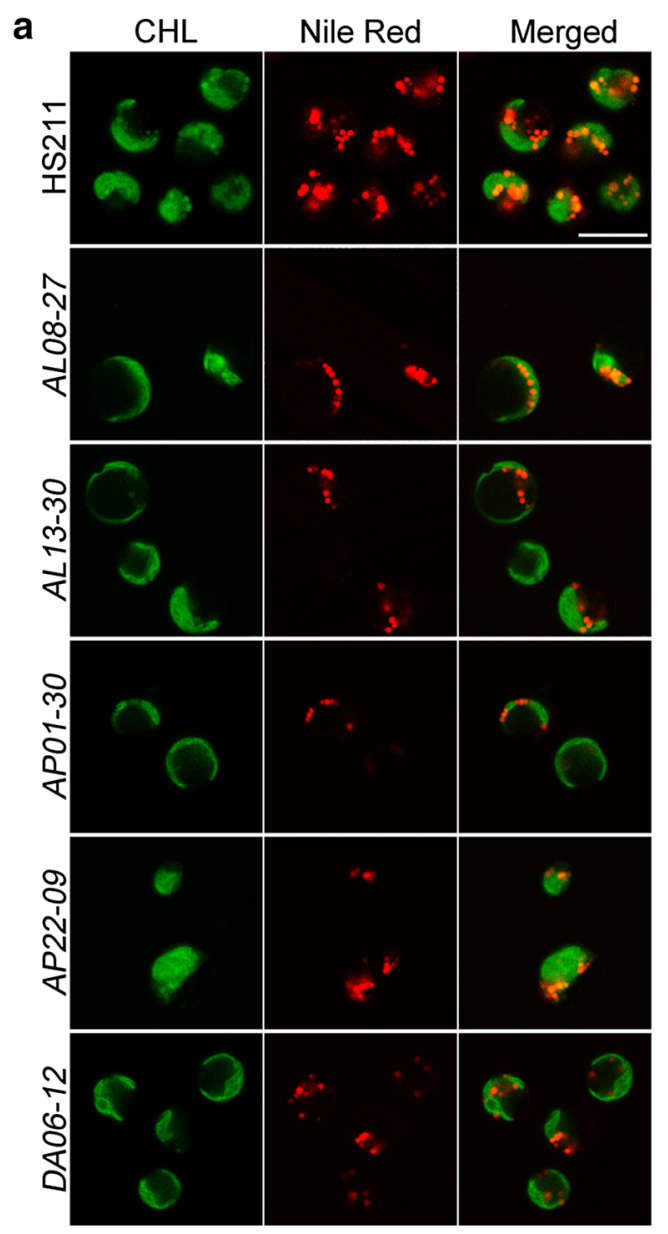

b
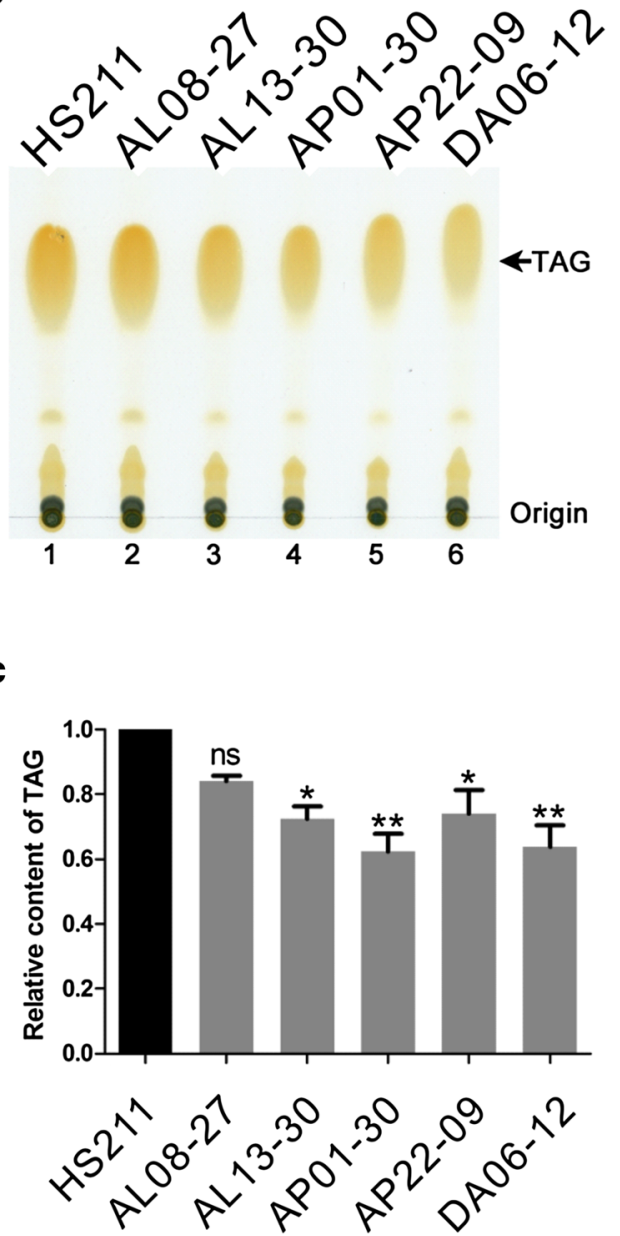

Fig. 4 Mutants with defects in oil droplet formation and TAG synthesis. a Nile red staining of oil droplets in the mutants and HS211 after 3-day nitrogen starvation. Scale bars $10 \mu \mathrm{m}$. CHL chlorophyll fluorescence. b Analysis of TAG biosynthesis in mutants with defective oil droplet formation. c The relative amount of TAG in the mutants as compared to that in WT. ${ }^{*} p<0.05 ;{ }^{* *} p<0.01$ 
possessing defects in oil droplet formation have been identified in this study, which is a great resource to study the molecular mechanism for assembly and disassembly of oil droplets.

\section{Characterization of mutants for targeted genes}

Initially, we planned to screen 61 mutants for genes involved in flagellar assembly, biogenesis of basal body, and vesicles trafficking (Additional file 2: Table S1). We identified 65 correct insertion loci corresponding to 37 genes from the super pool library during 6 months. Due to contamination and low recovery rate of certain transformants, only 48 insertion loci could be amplified in the basic pool. Finally, 26 mutants corresponding to 20 target genes were identified, including transition zone mutant $P O C 2$, flagella mutant $P K D 2$, glycosylation mutant MAN1, and starch metabolism mutant SSS2 (Fig. 5a; Table 2). Among them, 31\% insertion cassettes were integrated in the UTR, $23 \%$ were integrated in exons, and approximately $46 \%$ of insertions occurred in the introns (Fig. 5b). To determine whether mutagenesis in introns could induce complete knockout of target genes in Chlamydomonas, we compared the mRNA level of target genes in three individual mutants with parental strain HS211 by quantitative PCR, with probes locating at upstream, downstream, or across the insertion loci. The transcriptions of the three targeted genes were still detectable in the mutants. However, the transcript fragments upstream of the insertion loci (inserted in 13th intron) in mutant fap 215 were up-regulated. In contrast, the transcript across the insertion loci in mutant fap 215 and poc2 (inserted in 2nd intron) was undetectable. The transcription downstream of the insertion loci was variable, which was down-regulated in mutant fap164 (inserted in 2nd intron), but up-regulated by 20 -fold in mutant poc2 (Fig. 5c). Partial transcription was still detectable in the three mutants, which suggests that the 2.6-kb MlyI-digested expression cassette did not always terminate gene transcription completely when it was inserted into the intron. Therefore, another optimized insertion cassette was designed.

\section{Optimizing the insert insertion cassette}

Although the 2.6-kb MlyI-digested fragment contains two inverted terminators at its right border, it lacks a terminator on the left side. Additionally, terminators may not be functional for the target gene if the insertion cassette was located within an intron. To overcome this, we designed a new insertion cassette with a splicing donor sequence from the RubisCO gene (TCCATTTGCAG/GATGTTCGA) at both sides of the insert fragment. Thus, the insertion cassette became a part of the mRNA even if it was integrated into an intron. We also added three tandem overlapping stop codons and a transcription termination signal at each side (Fig. 6a). As a result, transcription of a target gene would terminate at

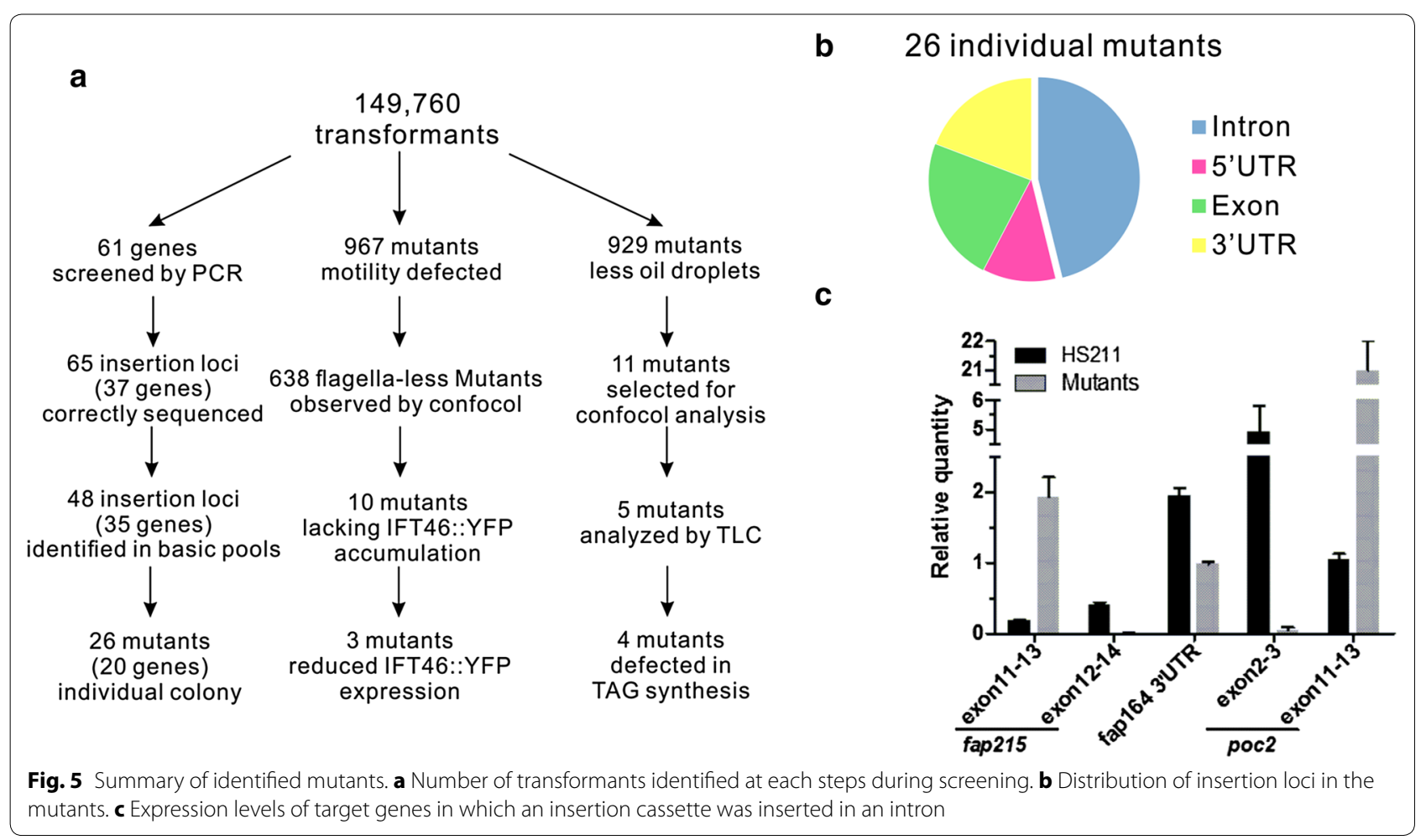


Table 2 List of identified mutants

\begin{tabular}{|c|c|c|c|c|}
\hline Targetedgenes & Accession $^{a}$ & Description & Function category & Insertion locib \\
\hline POC2 & Cre10.g436000 & Polycystin-related & Transition zone & $R, 2$ nd intron; $F$, 1st exon \\
\hline$C R X$ & Cre03.g202950 & $\begin{array}{l}\text { Ef-hand calcium-binding domain containing } \\
\text { protein }\end{array}$ & Calredoxin & $\mathrm{R}$, 2nd intron \\
\hline MAN1 & Cre07.g336600 & Alpha-1,2-mannosidase & Glycosylation & $\mathrm{R}, 7$ th exon \\
\hline CUL3 & Cre17.g734400 & Ubiquitin ligase SCF complex subunit Cullin & Ubiquitin ligase & $\mathrm{R}, 3^{\prime} \cup T R$ \\
\hline$X Y L T$ & Cre09.g391282 & Glycoprotein 2-beta-D-xylosyltransferase & Glycosylation & $F$, 6th intron; $F$, 7th intron \\
\hline PKD2 & Cre17.g715300 & Polycystin cation channel protein 2 & Flagellar protein & F, 11th exon; F, 3rd exon \\
\hline$A M Y B 1$ & Cre06.g307150 & Beta-amylase & Starch metabolism & R, 5'UTR; R, 3'UTR (two different mutants) \\
\hline SSS2 & Cre03.g185250 & Soluble starch synthase II & Starch metabolism & $\mathrm{R}$, 1st intron \\
\hline FAP215 & Cre03.g180450 & $\begin{array}{l}5^{\prime} \text {-nucleotidase and flagellar associated } \\
\text { protein }\end{array}$ & Flagellar protein & $\mathrm{R}, 13$ th intron \\
\hline FAP208 & Cre11.g482001 & Ankyrin repeat-containing protein & Flagellar protein & F, 22nd exon \\
\hline FAP249 & Cre17.g746697 & Flagellar associated protein & Flagellar protein & $\begin{array}{l}\text { R, } 5^{\prime} \text { UTR (ligated with } 480 \text { bp unknown } \\
\text { sequence) }\end{array}$ \\
\hline FAP164 & Cre17.g735350 & Flagellar associated protein & Flagellar protein & $\mathrm{F}$, 2nd intron \\
\hline CDPK3 & Cre01.g009500 & Protein kinase domain (Pkinase) & Flagellar protein & $\begin{array}{l}\text { F, 2nd intron (ligated with partial left border of } \\
\text { insertion fragments) }\end{array}$ \\
\hline PDCD6 & Cre03.g200050 & $\begin{array}{l}\text { Ca2+ -binding protein, EF-hand protein } \\
\text { superfamily }\end{array}$ & ESCRT & $\mathrm{R}$, 2nd intron; $\mathrm{R}$, 4th intron \\
\hline VPS4 & Cre02.g079300 & AAA-ATPase of VPS4/SKD1 family & ESCRT & $\mathrm{R}, 3^{\prime}$ UTR \\
\hline VPS37 & Cre08.g362550 & Subunit of the ESCRT-I complex & ESCRT & $\mathrm{F}$, 2nd intron \\
\hline PEPC2 & Cre03.g171950 & Phosphoenolpyruvate carboxylase & Lipid metabolism & R, 19th exon \\
\hline IFT139 & Cre06.g268800 & Intraflagellar transport protein 139 & $\mathrm{IFT}$ & $\mathrm{R}, 22$ nd intron \\
\hline UBA1 & Cre09.g386400 & Ubiquitin-activating enzyme E1 & Ubiquitin & $\mathrm{R}, 3^{\prime} \mathrm{UTR}$ \\
\hline FRE2 & Cre05.g241400 & Ferric-chelate reductase (NADH) & Ferric protein & $\mathrm{R}, 5^{\prime}$ UTR \\
\hline
\end{tabular}

${ }^{a}$ Gene accession numbers were from Phytozome v10.3 C. reinhardtii website

${ }^{\mathrm{b}} \mathrm{F}$ and $\mathrm{R}$ indicate that the insertion cassettes have the same or opposite orientation as the targeted genes, respectively

the artificial transcription termination signal and polyadenylated at the new terminal. Thus, transcription and translation would stop simultaneously. We transformed the modified aphVIIr fragment into the strain HS211, and found that more than twofold mutants with motility defects were generated under the same conditions as compared to the original aphVII fragment without splicing donor segments (Fig. 6b). We also simulated gene interruption by inserting different cassettes into the $\mathrm{RbcS} 2$ intron3 which was integrated to luciferase, and analyzed the luciferase activity of Chlamydomonas transformants in order to compare the termination efficiency of the new insertion cassette with or without splicing donor sequences (Additional file 4: Fig. S2). Luciferase interrupted by insertion cassettes possessing splicing donor sequence displayed relatively lower activity, with an average intensity of $\sim 3000-7300$ in pHK601-5, pHK603-5 and pHK603-10 compared to 13,000 in cassettes pHK602-2 which has no splicing donor sequence (Fig. 6c). Furthermore, insertion cassettes with splicing donor sequence generated less positive luciferase transformants. Only 2.5 and $3.9 \%$ positive transformants could be detected in pHK601-5 and pHK603-10, compared to $7.2 \%$ in pHK602-2. Transformant displaying high luciferase activity $\left(>10^{4}\right)$ could be hardly detected in pHK6015, pHK603-5 and pHK603-10 (Fig. 6d).

These results suggest that the new insertion cassette increases the efficiency of transcription termination, subsequently increasing the possibility of generating the null mutant.

\section{Discussion}

Due to a lack of reliable targeted gene editing methods such as TALEN or CRISPR-Cas9, large-scale insertion mutagenesis provides an alternative strategy to screen mutants of specific genes in Chlamydomonas. In this study, we generated and maintained an insertional mutagenesis library including approximately 150,000 clones, which can be utilized for both, reverse and forward genetics. A total of 26 mutants corresponding to 20 interested genes, related to glycosylation, starch metabolism, lipid metabolism, and flagellar proteins were identified by PCR-based screening. Meanwhile, two independent libraries related to flagellar assembly 


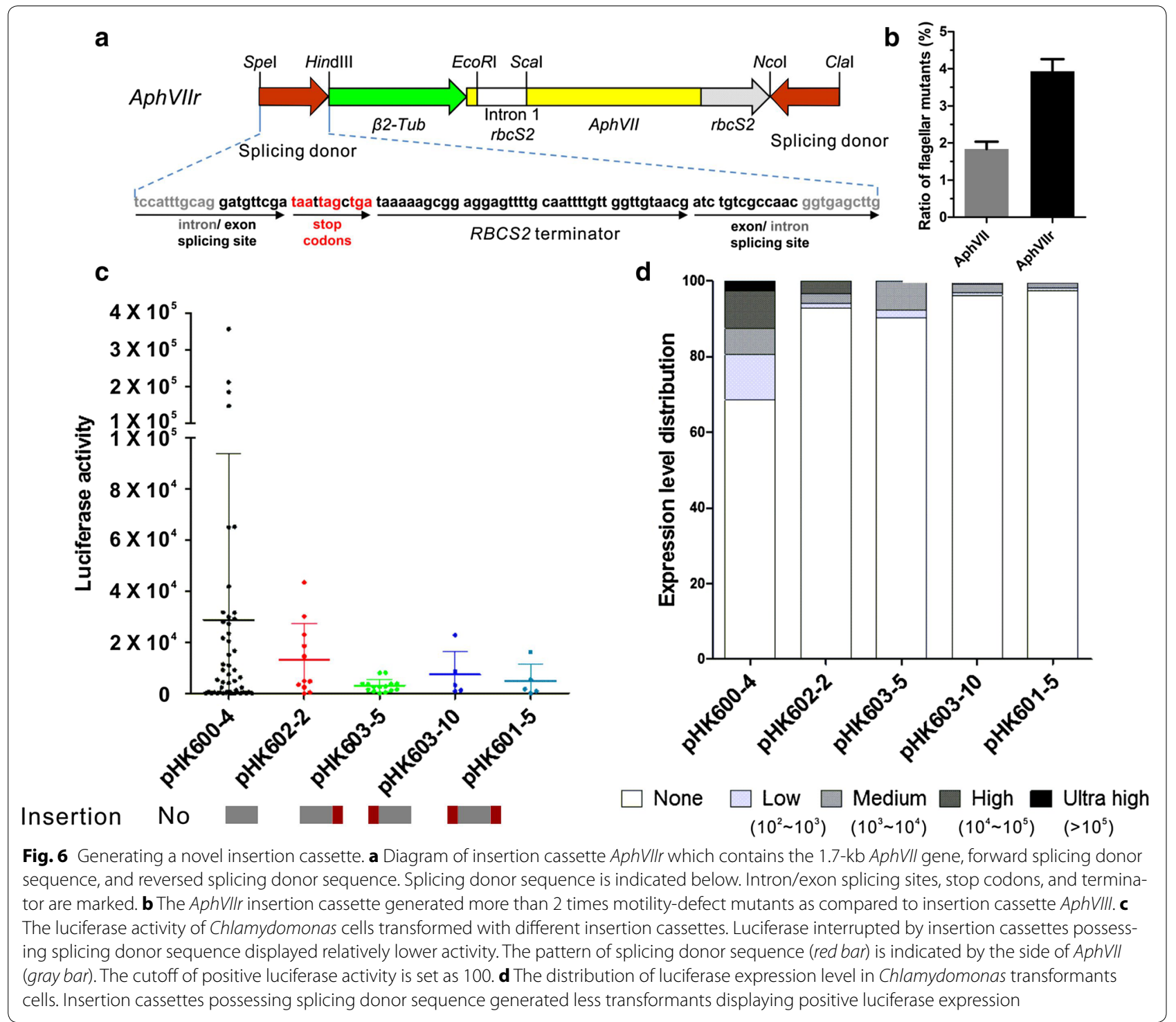

and oil droplet formation were obtained from this insertional library based on phenotype screening. The flagellar assembly mutant library included 967 mutants, which was expected to cover most of the $\sim 1000$ flagellar proteins $[52,53]$.

One advantage of this insertional library is that we transformed a fluorescence-labeled marker IFT46::YFP into the parental strain, which enabled us to screen specific mutants with defects in IFT. We obtained three mutants with reduced IFT46 expression in whole cells but lacking the accumulation at the basal body. The insertion loci of these mutants were identified and the interrupted genes may be involved in transporting or anchoring the IFT complex to the basal body. We also optimized several procedures for library construction and screening. First, the parental strain HS211 not only contains fluorescence
IFT marker but also displays higher transformation efficiency. We also noticed that a strain expressing the aphVIII gene showed 4.5-fold higher efficiency than wild type if another selection marker such as aphVII was used. This indicates that Chlamydomonas tends to acquire a second antibiotic resistance more easily if an antibiotic resistance gene is already expressed. The molecular mechanism of this phenomena still need to be identified, but it speeded library construction by reducing the number of transformation. Second, we optimized the insertion cassette specific primer, which complement to the junction between two inverted terminator, and increased the specificity for the PCR screening. Third, we maintained all the transformants on the 9-cm sized agar plate with a fixed 48-clones format. This format ensures much more cells to be maintained for replication and further analyses. Because 
there is more space between each colony, the cross contamination rates were reduced. However, the procedure of picking fresh transformants into 48-clones format is labor-intensive and cryopreservation of the whole library in liquid nitrogen is recommended to prevent mutant loss. Finally, we normalized the amount of genomic DNA of each transformant by picking the same amount of each individual clones from solid agar plates to make the DNA mixture, which increase the chance to identify the insertion junction of mutants with similar PCR efficiency.

Interestingly, we found that the insertion cassette was inserted in UTR region among 30\% mutants. The expression level of target gene was significantly reduced, which may be useful to screen the mutant of lethal genes. In addition, $46 \%$ of the insertions were localized in an intron. Their effect on the expression of the targeted gene varied. Hochmal et al., reported mutant IMcrx, in which an insertion cassette was integrated in the second intron, displayed the same phenotype as the miRNA knock-down strain. However, minor calredoxin (CRX) expression was still detectable in the mutant induced by high-intensity light [54]. For mutants such as, poc2-1, in which the insertion is in an intron, partial of mRNA expression was detected by PCR. And this phenomenon is also reported in the one-off library transformed with a shorter PCR-fragment [29]. It is possible that the entire insertion cassette is spliced with the flanking intron sequence at the exon/intron boundaries, which results in the expression of an entire mRNA at relatively lower efficiency. Therefore, the effect of insertion in an intron on the expression of the targeted gene should be determined case by case. In order to improve the knock out efficiency and reduce the mRNA expression of the targeted gene, we designed a new cassette with splicing donor sequence and tandem overlapping stop codon at both sides, which is supposed to terminate the gene transcription immediately when it was inserted in an intron or UTR region.

In summary, our insertional mutant library provides an alternative to obtain mutants of genes of interest for the Chlamydomonas research community. Additionally, the sub-library of flagella and oil droplet assembly mutants will serve as a useful resource to investigate the molecular mechanism of flagellar assembly and oil droplet formation.

\section{Conclusions}

In this study, we described comprehensive and economic methods to build an indexed mutant library including $\sim 150,000$ C. reinhardtii insertional mutants, which can be utilized for both reverse and forward genetics analysis. Using PCR-based screening method, we obtained 26 individual mutants corresponding to 20 different genes. Then, we identified 967 motility-defect mutants, including 10 mutants lacking IFT accumulation at the basal body. We also obtained 929 mutants defective in oil droplet assembly after nitrogen deprivation. In addition, a new insertion cassette was constructed to terminate the transcription of the targeted gene when the insertion cassette was inserted in introns and UTR. This library provides versatile resources for the research community to obtain Chlamydomonas mutants of any genes or with specific phenotype.

\section{Methods}

\section{Algal strains and growth conditions}

The flagella-less $C$. reinhardtii mutant and the wild type CC125 were kindly provided by the Chlamydomonas Resource Center. HS211 was used as parental strain for library construction. HS211 is a rescue strain of mutant ift46-1 obtained by transforming a linearized plasmid pHK266, which contains the IFT46 full length fused to the $5^{\prime}$ end of YFP (see "DNA manipulation" for details). Unless otherwise indicated, algal cells were grown mixtrophically in liquid TAP medium or on solid TAP agar (1.5\%) plate under continuous illumination $\left(30 \mu \mathrm{E} \mathrm{m}^{-2} \mathrm{~s}^{-1}\right)$ at $22{ }^{\circ} \mathrm{C}$. For maintenance of the library, algal colonies were grown under weak illumination $\left(5 \mu \mathrm{E} \mathrm{m}^{-2} \mathrm{~s}^{-1}\right)$ at $20^{\circ} \mathrm{C}$. For motility defect and oil droplet defect screening, algal colonies were inoculated into liquid TAP with omission of $\mathrm{NH}_{4} \mathrm{Cl}$.

\section{DNA manipulation}

The universal plasmid pHK86 includes fused GFP::YFP sequences driven by the $P S A D$ promoter, and the selectable marker gene AphVIII driven by HSP70A promoter [55]. For mutant ift46 rescue, the genomic DNA of IFT46 gene was amplified by PCR from the plasmid pGEM T Easy-IFT46 (provided by Joel Rosenbaum) using primers IFT46-F and IFT46-R (all primers listed in Additional file 3: Table S2). After digestion with NdeI and EcoRV, the IFT46 fragment was ligated to the $5^{\prime}$ side of YFP in plasmid pHK86, yielding the plasmid pHK214. The AphVIII in pHK214 were exchanged with the AphVII fragment amplified from the plasmid pHyg3 (provided by Wolfgang Mages), which yielded the plasmid pHK266. The plasmid pHK266 was linearized by KpnI and used to rescue the mutant ift46.

For library transformation, the 2.6-kb insertion cassette of AphVIII was digested from the plasmid pMJ013b with MlyI [27].

For optimization of the insertion cassette, the forward-splicing donor (FSD) sequence was synthesized by annealing the oligonucleotides F-Spe-F, F-Spe-R, F-Hind-F, and F-Hind-R, and the reversed one (RSD) was synthesized by annealing the oligonucleotides R-Nco-F, R-Nco-R, R-Cla-F, and R-Cla-R. The annealing process 
followed was as previously described by $\mathrm{Hu}$ et al. [38] After digestion with SpeI/HindIII or NcoI/ClaI, the forward and reverse splicing donor sequences were ligated to the 5'- and 3'-ends of AphVII, respectively, using pBluescript II KS as the backbone, which yielded the plasmid pHK415. The 1.9-kb fragment FSD::AphVII::RSD digested from $\mathrm{pHK} 415$ by $\mathrm{SpeI} / \mathrm{ClaI}$ was used for transformation.

The RbcS2 intron3 was ligated to the CTGGAG/ GTGCTG of luciferase using In-Fusion kit (Clontech). Different version of insertion cassettes were amplified from pHK415 and ligated to the restriction site of ApaI in $R b c S 2$ intron3.

\section{Nuclear transformation of Chlamydomonas reinhardtii}

Nuclear transformations of $C$. reinhardtii were performed by electroporation as previously described by Huang et al. [56]. In brief, algae cells were grown in $200 \mathrm{ml}$ TAP medium until the cell density reached $6 \times 10^{6}$ cells $/ \mathrm{ml}$. The cells were concentrated to $2 \times 10^{8}$ cells $/ \mathrm{ml}$ with prechilled TAP, supplemented with $60 \mathrm{mM}$ Sorbitol. Then, $250 \mu \mathrm{l}$ concentrated cells were mixed with $30 \mathrm{ng}$ DNA fragment in a 4-mm electroporation gap cuvette at $4{ }^{\circ} \mathrm{C}$. Electroporation was performed using ECM630 electro cell manipulator (BTX Harvard Apparatus) with following parameters: voltage, $800 \mathrm{~V}$; resistor, $1575 \Omega$; and capacitor, $50 \mu \mathrm{F}$. Transformants were recovered by incubation in $10 \mathrm{ml}$ liquid TAP (60 mM sorbitol) for $14 \mathrm{~h}$ under low illumination $\left(5 \mu \mathrm{E} \mathrm{m} \mathrm{m}^{-2} \mathrm{~s}^{-1}\right)$. The cells were sedimented by centrifugation at $2500 \mathrm{rpm}$ for $3 \mathrm{~min}$ and the pellet was mixed with $1 \mathrm{ml} 20 \%$ starch and positive colonies were screened on solid TAP agar plates containing $10 \mu \mathrm{g} / \mathrm{ml}$ paromomycin or $12.5 \mu \mathrm{g} / \mathrm{ml}$ hygromycin B.

\section{DNA and RNA extraction}

Total genomic DNA of Chlamydomonas was extracted using standard $\mathrm{CTAB} / \mathrm{phenol} / \mathrm{chloroform}$ method as previously described by Huang et al. [48].The DNA concentration of each super library was determined using HindIII digested lambda DNA (ThermoFisher Scientific, USA) as control and diluted to $100 \mathrm{ng} / \mu \mathrm{l}$ for PCR. Total RNA of Chlamydomonas was isolated using TRIzol $^{\circledR}$ reagent (ThermoFisher Scientific, USA) according to the manufacturer protocol. RNA concentration was determined by Quawell 5000 (Quawell Technology, USA). Residual genomic DNA in total RNA was removed by DNase I (RNase-free) (ThermoFisher Scientific, USA) before reverse transcription.

\section{PCR procedures}

For PCR-based screening, the target gene specific primers were designed using Primer Premier 5.0 software with following parameters: length, $20-26 \mathrm{bp}$; Tm,
58-62 ${ }^{\circ} \mathrm{C}$; GC\%, 40-60\% (all primers listed in Additional file 3: Table S2). PCR was performed in a $20-\mu$ l solution including $0.25 \mu \mathrm{M}$ target gene specific primer, $0.125 \mu \mathrm{M}$ insertion cassette specific primer LGR06-F, $0.2 \mathrm{mM}$ dNTP, $0.5 \mathrm{M}$ betaine (Sigma), 3\% DMSO (Amresco), 0.5 unit TransTaq DNA polymerase (HiFi) (Transgen, China) and 100 ng genomic DNA templates. The PCR program for super library screening was as follows, pre-incubation at $95^{\circ} \mathrm{C}$ for $5 \mathrm{~min}, 34$ cycles of denaturation at $95^{\circ} \mathrm{C}$ for $30 \mathrm{~s}$, annealing at $62{ }^{\circ} \mathrm{C}$ for $30 \mathrm{~s}$, and amplification at $72{ }^{\circ} \mathrm{C}$ for $2 \mathrm{~min}$. The colony PCR was performed according to the method previously described by Cao et al. [57].

For mRNA expression quantification, reverse transcription of RNA was performed with oligo-dT primers using First Strand cDNA Synthesis Kit (ThermoFisher Scientific, USA) according to the manufacturer instructions. Quantitative real-time PCR was performed by ABI 7900HT Fast Real-Time PCR System (Applied Biosystems) with SYBR Green Realtime PCR Master Mix (TOYOBO, Japan) according to the manufacturer instructions. Using housekeeping gene Chlamydomonas $\beta$ subunit-like polypeptide (CBLP, Cre06.g278222) as an internal control [58], the expression of other genes was quantified using standard curve assay in SDS 2.4 software (Applied Biosystems). All primers used in real-time PCR are listed in Additional file 3: Table S2.

For flanking sequence identification, RESDA-PCR was employed [59]. Degenerated primers (DegAluI, DegPstI, DegSacII, and DegTaqI) and primer Q0 were the same as the original method. Primers For1, For2, Rev1, and Rev2 were used as specific primers.

\section{Protein extraction and western blot analyses}

Whole-cell protein extraction and concentration were performed as previously described by $\mathrm{Hu}$ et al. [38] Whole cell proteins $(5 \mu \mathrm{g})$ were separated by $10 \%$ sodium dodecyl sulfate-polyacrylamide gel electrophoresis and transferred to $0.45-\mu \mathrm{m}$ nitrocellulose membranes (BioRad, USA) at $300 \mathrm{~mA}$ for $3 \mathrm{~h}$. After blocking with 5\% non-fat milk, the nitrocellulose membranes were probed with rabbit anti-IFT46 antibody (1:5000, immunized with the $N$-terminal 20 amino-acid peptide by Genscript) or mouse anti- $\alpha$-tubulin antibody $(1: 200,000$, \#T9026, Sigma). Immunodetection of IFT46 was detected by ECL (Millipore) with goat anti-rabbit secondary antibody (1:5000, \#6154, Sigma), and $\alpha$-tubulin was detected with goat anti-mouse secondary antibody (1:5000, \#A4416, Sigma).

\section{Extraction of total cellular lipids and thin-layer chromatography}

Extraction of total cellular lipids was performed as previously described by Bligh and Dyer [60] with minor 
modifications. In brief, cells (approximately $3 \times 10^{7}$ cells) were harvested by centrifugation at $6000 \mathrm{~g}$ at room temperature for $5 \mathrm{~min}$. The pellets were mixed with $400 \mu \mathrm{l}$ methanol:chloroform $(1: 1, \mathrm{v} / \mathrm{v})$ and vortexed for $5 \mathrm{~min}$. The suspension was mixed with $120 \mu \mathrm{l}$ PAK buffer $(1 \mathrm{M}$ $\mathrm{KCl}, 0.2 \mathrm{M}$ phosphate acid). The mixture was centrifuged at 12,000 $\mathrm{g}$ for $5 \mathrm{~min}$ and the lower chloroform phase was transferred to a new glass tube. Finally, additional chloroform was added into the organic phase until the constant volume was $20 \mu \mathrm{l}$.

For thin-layer chromatography, equal volume of total cellular lipid extract was loaded as a spot on $10 \times 20 \mathrm{~cm}$ silica gel GF254 TLC plates (Haiyang, China). Neutral lipids in the samples were separated using a hexane/ diethylether $(3 / 1, \mathrm{v} / \mathrm{v})$ solvent mixture. Then, the silica gel plates were dried under the hood and stained by iodine vapor at $37^{\circ} \mathrm{C}$ for $10 \mathrm{~min}$. Finally, the triacylglycerol bands were visualized by CanoScan 9000F (Canon, Japan) and analyzed by Quantity One software. All data were normalized to positive control.

\section{Microscopy}

Motility-deficient mutants were identified using stereomicroscopy (Nikon SMZ1500, Japan). Mutants with changes in IFT46::YFP basal body localization were screened using LSM710 confocal microscope (Carl Zeiss, Germany) (excitation: $514 \mathrm{~nm}$; emission: 519-560 nm). To screen oil droplet accumulation-deficient mutants, cells were stained in $0.1 \mu \mathrm{g} / \mathrm{ml}$ Nile red (Sigma, USA) for $15 \mathrm{~min}$ in the dark at $37^{\circ} \mathrm{C}$ [61]. Cells with fewer oil droplets were identified using ECLIPSE Ti-U fluorescence microscope (Nikon, Japan) (excitation: 465-495 nm; dichroic mirror: $505 \mathrm{~nm}$; emission: 515-555 nm).

Live cell images were captured using Leica SP8 confocal microscope. For Nile red, the excitation was $488 \mathrm{~nm}$ and the emission was 560-590 nm. For chlorophyll fluorescence (CHL), the excitation was $488 \mathrm{~nm}$ and the emission was $650-700 \mathrm{~nm}$.

\section{Measurement of flagellar length}

Chlamydomonas cells were fixed in $0.5 \%$ Lugol' s iodine solution and examined with differential interference contrast (DIC) microscopy using ECLIPSE Ti-U fluorescence microscope (Nikon, Japan) equipped with a $40 \times$ objective. Lengths of flagella were measured with NIS-Elements BR software. For each sample, at least 300 flagella were measured.

\section{Luciferase activity assay}

The luciferase activity was analyzed using the Renilla Luciferase Assay System (Promega, TM055) in 96 well microtiter plates according to the manual, with the modification that the algae cells were ruptured with glass-beads by vortex for $15 \mathrm{~min}$.

\section{Additional files}

Additional file 1: Figure S1. Comparison of the specificity of primer LGL03 and LGR06. More than sixteen non-specific PCR products were obtained with primer LGL03, whereas only four non-specific bands were obtained using primer LGR06.

Additional file 2: Table S1. List of genes for insertional mutants screening and PCR identified insertion loci from super libraries.

Additional file 3: Table S2. List of primers used in plasmid construction, RESDA-PCR, Realtime-PCR, and library screening.

Additional file 4: Figure S2. Different version of insertion cassettes. The 238 bp intron 3 of $R b c S 2$ was fused to the luciferase at the site of CTGGAG/ GTGCTG. The insertion cassette with or without splicing donor sequence at either side of AphVII gene was inserted to the restriction site of Apal in RbcS2 intron3.

\section{Abbreviations}

CRISPR-Cas9: clustered regularly interspaced short palindromic repeats and CRISPR-associated protein 9; IFT: intraflagellar transport; EMS: ethyl methanesulfonate; MNNG: $N$-methyl- $N^{\prime}$-nitro- $N$-nitrosoguanidine; HR: homologous recombination; JGI: joint genome institute; RESDA-PCR: restriction enzyme site-directed amplification; TAP-N: nitrogen-deprived TAP medium; TLC: thin layer chromatography; TAG: triacylglycerol; FSD: forward-splicing donor; RSD: reverse-splicing donor.

\section{Authors' contributions}

KH organized the project. XC, GL, WK, LZ, BL, XM, NX, XX, XD performed the experiments, and analyzed the data. $\mathrm{KH}, \mathrm{GL}, \mathrm{XC}$ wrote the paper. All authors read and approved the final manuscript.

\section{Author details}

${ }^{1}$ Key Laboratory of Algal Biology, Institute of Hydrobiology, Chinese Academy of Sciences, Wuhan 430072, China. ${ }^{2}$ University of Chinese Academy of Sciences, Beijing 100039, China. ${ }^{3}$ College of Life Sciences, Hubei University, Wuhan 430062, China.

\section{Acknowledgements}

We would like to thank Dr. Jue Ning (University of Texas Southwestern) for suggestion on PCR solution for super library screening.

\section{Competing interests}

The authors declare that they have no competing interests.

\section{Availability of data and materials}

All data generated or analyzed during this study are included in this published article and its supplementary information files, including supplemental Additional file 1: Figure S1, Additional file 2:Table S1 and Additional file 3: Table S2. The experiment materials generated during the current study are available from the corresponding author on reasonable request.

\section{Funding}

This project was supported by the National Natural Science Foundation of China (31371354 to Dr. Kaiyao Huang and 31400654 to Dr. Gai Liu).

\section{Publisher's Note}

Springer Nature remains neutral with regard to jurisdictional claims in published maps and institutional affiliations.

Received: 29 September 2016 Accepted: 2 May 2017

Published online: 15 May 2017 


\section{References}

1. Merchant SS, Prochnik SE, Vallon O, Harris EH, Karpowicz SJ, Witman GB, Terry A, Salamov A, Fritz-Laylin LK, Marechal-Drouard L, et al. The Chlamydomonas genome reveals the evolution of key animal and plant functions. Science. 2007;318(5848):245-50.

2. Harris EH. Chlamydomonas as a model organism. Annu Rev Plant Physiol Plant Mol Biol. 2001;52:363-406.

3. Goodenough U. Historical perspective on Chlamydomonas as a model for basic research: 1950-1970. Plant J. 2015:82(3):365-9.

4. Baldari CT, Rosenbaum J. Intraflagellar transport: it's not just for cilia anymore. Curr Opin Cell Biol. 2010:22(1):75-80.

5. Rasala BA, Mayfield SP. The microalga Chlamydomonas reinhardtii as a platform for the production of human protein therapeutics. Bioeng Bugs. 2011;2(1):50-4.

6. Merchant SS, Kropat J, Liu B, Shaw J, Warakanont J. TAG, you're it! Chlamydomonas as a reference organism for understanding algal triacylglycerol accumulation. Curr Opin Biotechnol. 2012;23(3):352-63.

7. Smith GM. A monograph of the algal genus Scenedesmus based upon pure culture studies. Madison: Wisconsin Academy of Sciences, Arts and Letters; 1916.

8. Levine RP. A screening technique for photosynthetic mutants in unicellular algae. Nature. 1960;188:339-40.

9. Loppes R. Ethyl methanesulfonate: an effective mutagen in Chlamydomonas reinhardi. Mol Gen Genet MGG. 1968;102(3):229-31.

10. Davies DR, Plaskitt A. Genetical and structural analyses of cell-wall formation in Chlamydomonas reinhardi. Genet Res. 1971;17(01):33-43.

11. Huang B, Rifkin MR, Luck DJ. Temperature-sensitive mutations affecting flagellar assembly and function in Chlamydomonas reinhardtii. J Cell Biol. 1977;72(1):67-85

12. Fork DC, Urbach W. Evidence for the localization of plastocyanin in the electron-transport chain of photosynthesis. Proc Natl Acad Sci USA. 1965;53(6):1307-15

13. Kindle KL. High-frequency nuclear transformation of Chlamydomonas reinhardtii. Proc Natl Acad Sci USA. 1990;87(3):1228-32.

14. Cole DG, Diener DR, Himelblau AL, Beech PL, Fuster JC, Rosenbaum JL. Chlamydomonas kinesin-II-dependent intraflagellar transport (IFT): IFT particles contain proteins required for ciliary assembly in Caenorhabditis elegans sensory neurons. J Cell Biol. 1998;141(4):993-1008.

15. Debuchy R, Purton S, Rochaix JD. The argininosuccinate lyase gene of Chlamydomonas reinhardtii: an important tool for nuclear transformation and for correlating the genetic and molecular maps of the ARG7 locus. EMBO J. 1989:8(10):2803-9.

16. Shimogawara K, Fujiwara S, Grossman A, Usuda H. High-efficiency transformation of Chlamydomonas reinhardtii by electroporation. Genetics. 1998;148(4):1821-8.

17. Kumar SV, Misquitta RW, Reddy VS, Rao BJ, Rajam MV. Genetic transformation of the green alga_Chlamydomonas reinhardtii by Agrobacterium tumefaciens. Plant Sci. 2004;166(3):731-8.

18. Boynton J, Gillham N, Harris E, Hosler J, Johnson A, Jones A, RandolphAnderson B, Robertson D, Klein T, Shark K, et al. Chloroplast transformation in Chlamydomonas with high velocity microprojectiles. Science. 1988;240(4858):1534-8

19. Randolph-Anderson BL, Boynton JE, Gillham NW, Harris EH, Johnson AM, Dorthu M-P, Matagne RF. Further characterization of the respiratory deficient dum-1 mutation of Chlamydomonas reinhardtii and its use as a recipient for mitochondrial transformation. Mol Gen Genet MGG. 1993:236(2):235-44

20. Kindle KL, Schnell RA, Fernandez E, Lefebvre PA. Stable nuclear transformation of Chlamydomonas using the Chlamydomonas gene for nitrate reductase. J Cell Biol. 1989;109(6 Pt 1):2589-601.

21. Sizova I, Fuhrmann M, Hegemann P. A Streptomyces rimosus aphVIII gene coding for a new type phosphotransferase provides stable antibiotic resistance to Chlamydomonas reinhardtii. Gene. 2001;277(1-2):221-9.

22. Berthold P, Schmitt R, Mages W. An engineered Streptomyces hygroscopicus aph 7" gene mediates dominant resistance against hygromycin B in Chlamydomonas reinhardtii. Protist. 2002;153(4):401-12.

23. Stevens DR, Rochaix JD, Purton S. The bacterial phleomycin resistance geneble as a dominant selectable marker in Chlamydomonas. Mol Gen Genet MGG. 1996;251(1):23-30.

24. Barahimipour R, Neupert J, Bock R. Efficient expression of nuclear transgenes in the green alga Chlamydomonas: synthesis of an HIV antigen and development of a new selectable marker. Plant Mol Biol. 2016;90(4-5):403-18.

25. Garcia-Echauri SA, Cardineau GA. TETX: a novel nuclear selection marker for Chlamydomonas reinhardtii transformation. Plant Methods. 2015;11:27.

26. Brueggeman AJ, Kuehler D, Weeks DP. Evaluation of three herbicide resistance genes for use in genetic transformations and for potential crop protection in algae production. Plant Biotechnol J. 2014;12(7):894-902.

27. Zhang R, Patena W, Armbruster U, Gang SS, Blum SR, Jonikas MC. High-throughput genotyping of green algal mutants reveals random distribution of mutagenic insertion sites and endonucleolytic cleavage of transforming DNA. Plant Cell. 2014;26(4):1398-409.

28. Dent RM, Sharifi MN, Malnoe A, Haglund C, Calderon RH, Wakao S, Niyogi KK. Large-scale insertional mutagenesis of Chlamydomonas supports phylogenomic functional prediction of photosynthetic genes and analysis of classical acetate-requiring mutants. Plant J. 2015;82(2):337-51.

29. Gonzalez-Ballester D, Pootakham W, Mus F, Yang W, Catalanotti C, Magneschi L, de Montaigu A, Higuera JJ, Prior M, Galvan A, et al. Reverse genetics in Chlamydomonas: a platform for isolating insertional mutants. Plant Methods. 2011;7:24

30. Dutcher SK, Li L, Lin H, Meyer L, Giddings TH Jr, Kwan AL, Lewis BL. Whole-genome sequencing to identify mutants and polymorphisms in Chlamydomonas reinhardtii. G3 (Bethesda). 2012;2(1):15-22.

31. Rymarquis LA, Handley JM, Thomas M, Stern DB. Beyond complementation. Map-based cloning in Chlamydomonas reinhardtii. Plant Physiol. 2005;137(2):557-66

32. Gonzalez-Ballester D, de Montaigu A, Higuera JJ, Galvan A, Fernandez E. Functional genomics of the regulation of the nitrate assimilation pathway in Chlamydomonas. Plant Physiol. 2005;137(2):522-33.

33. Terashima M, Freeman ES, Jinkerson RE, Jonikas MC. A fluorescenceactivated cell sorting-based strategy for rapid isolation of high-lipid Chlamydomonas mutants. Plant J. 2015:81(1):147-59.

34. Neupert J, Karcher D, Bock R. Generation of Chlamydomonas strains that efficiently express nuclear transgenes. Plant J. 2009:57(6):1140-50.

35. Barahimipour R, Strenkert D, Neupert J, Schroda M, Merchant SS, Bock R. Dissecting the contributions of GC content and codon usage to gene expression in the model alga Chlamydomonas reinhardtii. Plant J. 2015;84(4):704-17.

36. Rasala BA, Barrera DJ, Ng J, PlucinakTM, Rosenberg JN, Weeks DP, Oyler GA, Peterson TC, Haerizadeh F, Mayfield SP. Expanding the spectral palette of fluorescent proteins for the green microalga Chlamydomonas reinhardtii. Plant J. 2013;74(4):545-56.

37. Schroda M. RNA silencing in Chlamydomonas: mechanisms and tools Curr Genet. 2006;49(2):69-84.

38. Hu J, Deng X, Shao N, Wang G, Huang K. Rapid construction and screening of artificial microRNA systems in Chlamydomonas reinhardtii. Plant J. 2014;79(6):1052-64

39. Molnar A, Bassett A, Thuenemann E, Schwach F, Karkare S, Ossowski S, Weigel D, Baulcombe D. Highly specific gene silencing by artificial microRNAs in the unicellular alga Chlamydomonas reinhardtii. Plant J. 2009;58(1):165-74.

40. Ferrante P, Diener DR, Rosenbaum JL, Giuliano G. Nickel and low CO(2)controlled motility in Chlamydomonas through complementation of a paralyzed flagella mutant with chemically regulated promoters. BMC Plant Biol. 2011:11:22

41. Ohresser M, Matagne RF, Loppes R. Expression of the arylsulphatase reporter gene under the control of the nit1 promoter in Chlamydomonas reinhardtii. Curr Genet. 1997;31(3):264-71.

42. Sizova I, Greiner A, Awasthi M, Kateriya S, Hegemann P. Nuclear gene targeting in Chlamydomonas using engineered zinc-finger nucleases. Plant J. 2013;73(5):873-82.

43. Zorin B, Lu Y, Sizova I, Hegemann P. Nuclear gene targeting in Chlamydomonas as exemplified by disruption of the PHOT gene. Gene. 2009:432(1-2):91-6.

44. Baek K, Kim DH, Jeong J, Sim SJ, Melis A, Kim JS, Jin E, Bae S. DNA-free two-gene knockout in Chlamydomonas reinhardtii via CRISPR-Cas9 ribonucleoproteins. Sci Rep. 2016;6:30620.

45. Shin SE, Lim JM, Koh HG, Kim EK, Kang NK, Jeon S, Kwon S, Shin WS, Lee B, Hwangbo K, et al. CRISPR/Cas9-induced knockout and knock-in mutations in Chlamydomonas reinhardtii. Sci Rep. 2016:6:27810.

46. Jiang W, Brueggeman AJ, Horken KM, Plucinak TM, Weeks DP. Successful transient expression of Cas9 and single guide RNA genes in Chlamydomonas reinhardtii. Eukaryot Cell. 2014;13(11):1465-9. 
47. Li X, Zhang R, Patena W, Gang SS, Blum SR, Ivanova N, Yue R, Robertson JM, Lefebvre PA, Fitz-Gibbon ST, et al. An indexed, mapped mutant library enables reverse genetics studies of biological processes in Chlamydomonas reinhardtii. Plant Cell. 2016;28(2):367-87.

48. Huang K, Merkle T, Beck CF. Isolation and characterization of a Chlamydomonas gene that encodes a putative blue-light photoreceptor of the phototropin family. Physiol Plant. 2002;115(4):613-22.

49. Randall J. The flagellar apparatus as a model organelle for the study of growth and morphopoiesis. Proc R Soc Lond Ser B Biol Sci. 1030;1969(173):31-55.

50. Richey EA, Qin H. Dissecting the sequential assembly and localization of intraflagellar transport particle complex B in Chlamydomonas. PLoS ONE. 2012;7(8):e43118.

51. Scranton MA, Ostrand JT, Fields FJ, Mayfield SP. Chlamydomonas as a model for biofuels and bio-products production. Plant J. 2015;82(3):523-31.

52. Nguyen HM, Baudet M, Cuine S, Adriano JM, Barthe D, Billon E, Bruley C, Beisson F, Peltier G, Ferro M, et al. Proteomic profiling of oil bodies isolated from the unicellular green microalga Chlamydomonas reinhardtii: with focus on proteins involved in lipid metabolism. Proteomics. 2011;11(21):4266-73.

53. Pazour GJ, Agrin N, Leszyk J, Witman GB. Proteomic analysis of a eukaryotic cilium. J Cell Biol. 2005;170(1):103-13.
54. Hochmal AK, Zinzius K, Charoenwattanasatien R, Gabelein P, Mutoh R, Tanaka H, Schulze S, Liu G, Scholz M, Nordhues A, et al. Calredoxin represents a novel type of calcium-dependent sensor-responder connected to redox regulation in the chloroplast. Nat Commun. 2016;7:11847.

55. Long H, Huang K. Analysis of flagellar protein ubiquitination. Methods Enzymol. 2013;524:59-73.

56. Huang K, Diener DR, Mitchell A, Pazour GJ, Witman GB, Rosenbaum JL. Function and dynamics of PKD2 in Chlamydomonas reinhardtii flagella. J Cell Biol. 2007;179(3):501-14.

57. Cao M, Fu Y, Guo Y, Pan J. Chlamydomonas (Chlorophyceae) colony PCR. Protoplasma. 2009:235(1-4):107-10

58. Schloss JA. A Chlamydomonas gene encodes a $G$ protein beta subunitlike polypeptide. Mol Gen Genet MGG. 1990;221(3):443-52.

59. Gonzalez-Ballester D, de Montaigu A, Galvan A, Fernandez E. Restriction enzyme site-directed amplification PCR: a tool to identify regions flanking a marker DNA. Anal Biochem. 2005;340(2):330-5.

60. Bligh EG, Dyer WJ. A rapid method of total lipid extraction and purification. Can J Biochem Physiol. 1959;37(8):911-7.

61. Greenspan P, Mayer EP, Fowler SD. Nile red: a selective fluorescent stain for intracellular lipid droplets. J Cell Biol. 1985;100(3):965-73.

\section{Submit your next manuscript to BioMed Central and we will help you at every step:}

- We accept pre-submission inquiries

- Our selector tool helps you to find the most relevant journal

- We provide round the clock customer support

- Convenient online submission

- Thorough peer review

- Inclusion in PubMed and all major indexing services

- Maximum visibility for your research

Submit your manuscript at www.biomedcentral.com/submit 